\title{
Simulating Electron Dynamics in Open Quantum Systems under Magnetic Fields
}

\section{Supporting Information}

Tal Maaravi and Oded Hod*

\begin{abstract}
Department of Physical Chemistry, School of Chemistry, The Raymond and Beverly Sackler Faculty of Exact Sciences and The Sackler Center for Computational Molecular and Materials Science, Tel Aviv University, Tel Aviv 6997801,
\end{abstract}

Israel

\section{Driving Rate Sensitivity Test}

As mentioned in the main text, the driving rate appearing in Eq. (6) of the main text, can be evaluated from the properties of the implicit baths and their couplings to the finite lead models. ${ }^{1}$ Nevertheless, for the sake of simplicity, in the present study we opted to approximate the value of the driving rate by examining the lead level spacing within the Fermi transport window. The chosen value of $\Gamma \approx \frac{2 \Delta \varepsilon_{\max }}{\hbar} \approx 0.05 \mathrm{fs}^{-1}$ was extracted from the largest energy spacing within the transport window of $\Delta \varepsilon_{\max } \sim 0.016 \mathrm{eV}$. In Fig. S1a we plot the density of lead states for the 2500 atoms chain lead model using a Lorentzian broadening of $\hbar \Gamma$. When using the value of $\Gamma=0.05 \mathrm{fs}^{-1}$ (red curve) a relatively smooth DOS curve is obtained that indicates a good representation of the corresponding DOS of the semi-infinite chain, as required.

In principle, the simulation results should be insensitive to the specific choice of driving rate within a certain physically reasonable range. ${ }^{2-5}$ To check for this, we repeated our calculations for the $180^{\circ}$ connected system strongly coupled to 300 atom chains serving as EM lead portions (see main text for model details) that are coupled at their far end to 2500 atom chain leads, on which the boundary conditions are imposed using driving rates of $\Gamma=0.025 \mathrm{fs}^{-1}$ and $\Gamma=$ $0.1 \mathrm{fs}^{-1}$. Since the driving term is applied only to the lead sections this validity test should apply also to the $104^{\circ}$ connected system coupled to the same lead models. Examining first the lead DOS with these two broadening values we find that for the larger value of $\Gamma=0.1 \mathrm{fs}^{-1}$ the discrete nature of the finite lead model states vanishes, and the van Hove singularities near the band edges broaden, whereas for the lower value of $\Gamma=0.025 \mathrm{fs}^{-1}$ the individual lead states are clearly manifested in the central region of the DOS (see inset in Fig. S1a). The corresponding effect on the steady-state currents, obtained using the Sylvester equation (see Eq. (7) and appendix A of the main text), is shown in Fig. S1b as a function of normalized magnetic flux threading the ring. The steady-state currents obtained using the three driving rates considered present minor quantitative variations throughout the half-AB period considered with the same qualitative behavior. This indicates that our choice of driving rate value is within the stability region of the system. $2,4,5$

Nonetheless, since we are interested in time-dependent properties of Aharonov-Bohm type molecular junctions, we further verified that not only the steady-state currents but also the switching dynamics is insensitive to the choice of driving rate. To that end, we present in Fig. S2 the temporal current traces following the magnetic field switch-on for the three driving rate values considered at a fixed lead model size. The results clearly demonstrate that the three traces are in very good agreement thus indicating the validity of our choice of leads driving rate. It should be noted, that with the chosen $\Gamma$ values the leads are driven towards their respective equilibrium states at a rate that is of the order of the typical relaxation rate of the system. This, however, does not seem to strongly influence our ability to provide a reliable description of the systems dynamics. We attribute that to the presence of the extended molecule lead sections that are chosen to be sufficiently large to allow the system (ring) to relax even before the perturbation reaches the driven leads 
dissipative region. Hence, for the calculations presented in the main text we set the value of the driving rate to $\Gamma=$ $0.05 \mathrm{fs}^{-1}$ such that the finite lead model appropriately represents the electronic structure of its infinite counterpart while providing converged dynamical properties. We note that even better stability of the results with respect to the driving rate can be achieved by extracting state-dependent broadening factors from the properties of the implicit reservoirs and their couplings to the finite lead models. ${ }^{1}$

(a)

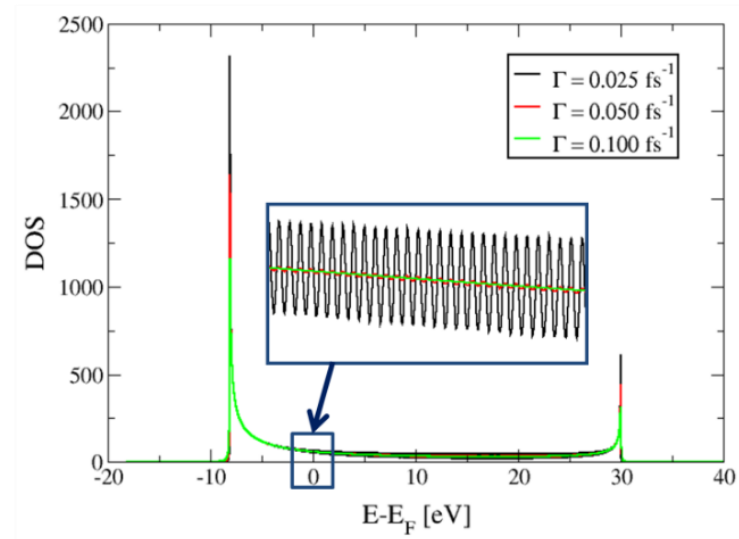

(b)

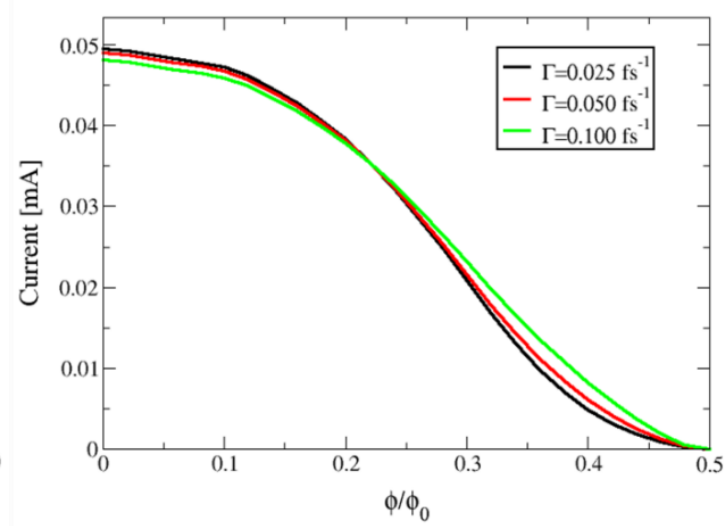

Figure S1: Driving rate sensitivity analysis. (a) Density of states of the 2500 hydrogen atoms lead model obtained with Lorentzian broadening of $\hbar \Gamma$ with $\Gamma=0.025 \mathrm{fs}^{-1}$ (black), $0.05 \mathrm{fs}^{-1}$ (red), and $0.1 \mathrm{fs}^{-1}$ (green). Zoom-in on the Fermi-window area is presented in the inset. (b) Steady-state current plotted against the normalized magnetic flux threading the $180^{\circ}$ connected ring that is strongly coupled to 300 hydrogen atom chains (see main text for model details) serving as EM lead portions that are strongly coupled at their far end to 2500 hydrogen chain lead models, calculated for the above mentioned driving rates (same color code as in panel $(a)$ ). The applied bias voltage of $V_{b}=$ $1 \mathrm{~V}$ and electronic temperature of $\mathrm{T}=300 \mathrm{~K}$ are the same as those used to obtain the results presented in the main text.

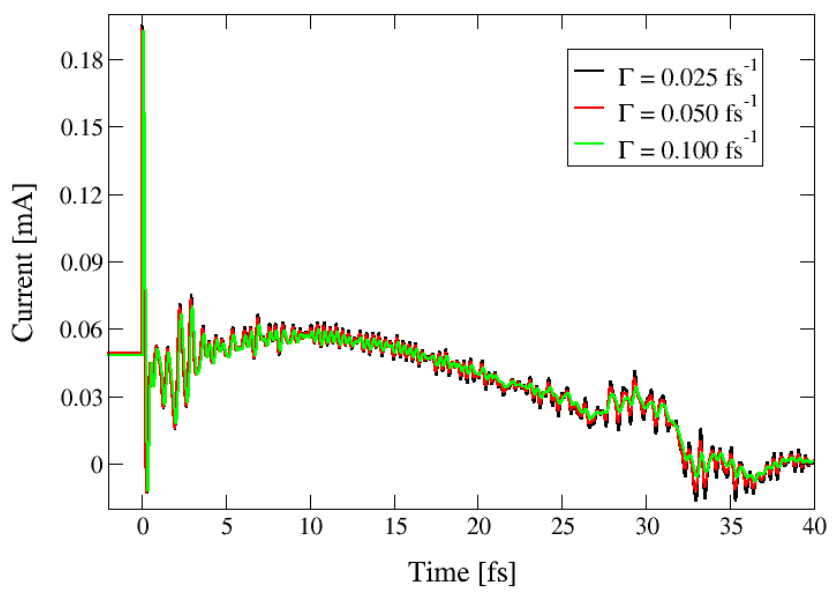

Figure S2: Driving rate sensitivity analysis for the real-time switching dynamics of the $180^{\circ}$ connected ring that is strongly coupled to 300 atom chains (see main text for model details) serving as EM lead portions that are strongly coupled at their far end to 2500 hydrogen atom chain lead models. At time $t=0 \mathrm{fs}$ a magnetic flux of $\phi=0.5 \phi_{0}$ is applied to a system that was at steady-state in the absence of the field and the current dynamics towards the new steadystate is calculated for $\Gamma=0.025 \mathrm{fs}^{-1}$ (black), $0.05 \mathrm{fs}^{-1}$ (red), and $0.1 \mathrm{fs}^{-1}$ (green). The applied bias voltage of $V_{b}=$ $1 \mathrm{~V}$ and electronic temperature of $T=300 \mathrm{~K}$ are the same as those used to obtain the results presented in the main text. 


\section{Convergence with respect to the Lead Model Size}

The Markovian approximation adopted in the driven Liouville von Neumann (DLvN) methodology requires the use of sufficiently large lead models, whose characteristic dynamic time-scale is larger than the typical memory relaxation time of the active molecule. In recent years, general criteria have been set for the lead model size required to assure validity of the Markovian approximation. ${ }^{2-5}$ Nevertheless, it is a good practice to verify that the simulation results are converged with respect to the chosen lead model size. This is especially true for weak coupling scenarios, where the lifetime of charge carriers on the active molecule is long. To that end, we repeated the steady-state calculations of the strongly coupled $180^{\circ}$ connected system and the weakly coupled $104^{\circ}$ connected system with various lead model sizes using the Sylvester equation (see Eq. (7) and appendix A in the main text) and the non-equilibrium Green's function (NEGF) scheme (see appendix $\mathrm{C}$ in the main text).

In the DLvN calculations of the strongly coupled $180^{\circ}$ system the two 300 atom chains that serve as extended molecule (EM) lead portions are further strongly coupled at their far end to hydrogen chain leads of sizes 1000, 2500, and 3000 atoms. We note that with increasing finite lead model size its density of states (DOS) increases and the coupling of each lead state to the implicit bath states decreases. ${ }^{6}$ Therefore, the value of the driving rate, which serves to broaden the various lead states due to their coupling to the implicit bath, should be decreased accordingly. Fig. S3 shows the resulting steady-state currents flowing through the ring as function of the normalized magnetic flux threading its cross section. When increasing the lead size from 1000 (full black line) to 1500 (full purple line) and 2500 (full red line) atoms, the agreement with the NEGF results (full blue line) improves both qualitatively and quantitatively. Further increasing the lead models size to 3000 atoms results in very little variation of the current (green circles).

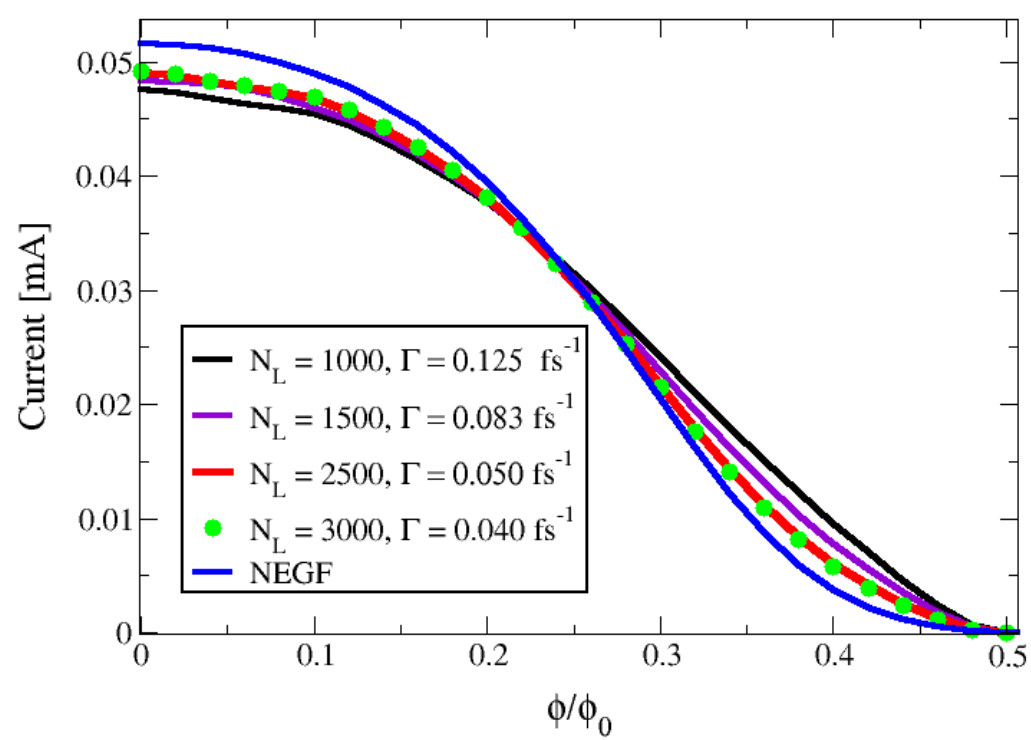

Figure S3: Strong coupling finite lead model size convergence test. Steady-state DLvN current plotted against the normalized magnetic flux threading the $180^{\circ}$ connected ring strongly coupled (see main text for the full model parameters) to two 300 hydrogen atom chains serving as EM lead portions that are strongly coupled at their far end to hydrogen chain lead models of sizes 1000 (full black line), 1500 (full purple line), 2500 (full red line), and 3000 (green circles) atoms, compared to the NEGF results (full blue line). The applied bias voltage of $V_{b}=1 \mathrm{~V}$ and electronic temperature of $T=300 \mathrm{~K}$ are the same as those used to obtain the results presented in the main text. 
A similar behavior is found for the weakly coupled $104^{\circ}$ connected system. In Fig. S4 we show that increasing the lead model size from 2500 (full black line) to 3000 (full red line) atoms improves the agreement to the NEGF results (full blue line). Further increasing the lead size to 3300 atoms (dashed green line) shows minor variations in the current-flux curve.

We therefore conclude that, for the purpose of the switching dynamics simulations presented in the main text, it is sufficient to use the 2500 hydrogen atom chain lead models. These provide both good qualitative and quantitative agreement with steady-state NEGF results, especially in the switch region of the weakly coupled system $\left(\frac{\phi}{\phi_{0}} \in\right.$ [0: 0.01]), with reasonable computational burden.

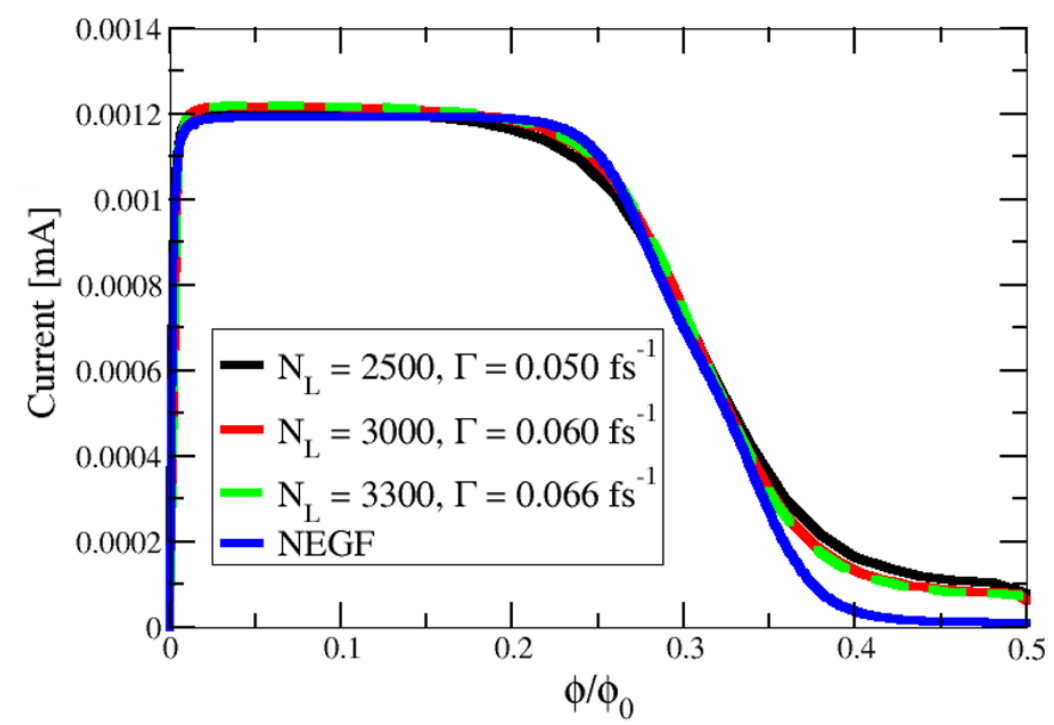

Figure S4: Finite lead model size convergence test in weakly coupled rings. Steady-state DLvN current plotted against the normalized magnetic flux threading the $104^{\circ}$ connected ring weakly coupled (see main text for the full model parameters) to hydrogen atom chain lead models of sizes 2500 (full black line), 3000 (full red line), 3300 (dashed green lines) atoms, compared to the NEGF results (full blue line). The applied bias voltage of $V_{b}=1 \mathrm{~V}$ and electronic temperature of $T=300 \mathrm{~K}$ are the same as those used to obtain the results presented in the main text. 


\section{Spectrum Bandwidth Cutoff Validity Test}

The current generated in the system is determined by the properties of the molecule, the leads, and their mutual coupling. The molecule sets the nature and density of conducting states; the leads dictate the potential bias drop across the system via the chemical potentials and electronic temperatures of the reservoirs to which they are connected; and their coupling determines the lifetime of charge carriers on the molecule and the position of the molecular states. As demonstrated above, the lead model size is chosen to provide a sufficiently dense manifold of states within the Fermi transport window such that convergence of the obtained current is achieved. Nevertheless, since the typical lead bandwidth is significantly larger than the bias drop across the system, the vast majority of lead states resides outside the Fermi transport window and therefore contributes negligibly to the conductance properties of the system. Hence, a considerable reduction of computational burden, with little effect on the calculated current, can be achieved by applying an energy cutoff on the leads and molecule spectra excluding from the transport calculation all levels that reside well outside the Fermi window. ${ }^{7}$ To this end, following the transformation of the full system Hamiltonian to the state representation, we extract from it a reduced dimension Hamiltonian that includes all energy levels of the leads and the extended molecule sections that reside within an energy window of $\left(E_{F}-\frac{1}{2} V_{b}-\right.$ $\left.W, E_{F}+\frac{1}{2} V_{b}+W\right)$. Here, $E_{F}$ is the Fermi energy of the entire finite model system, $V_{b}$ is the applied bias voltage, and $\mathrm{W}$ is the width of the buffer energy range set to obtain converged current values to within a desired accuracy. The latter depends on the reservoirs' electronic temperatures and on the leads-molecule couplings.

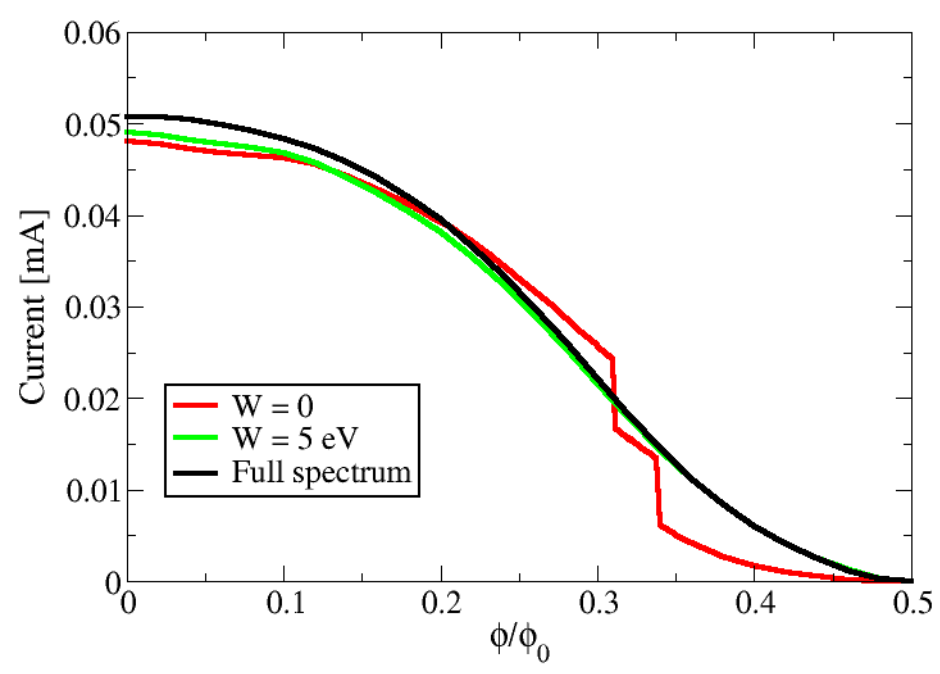

Figure S5: Spectrum cutoff convergence test. Steady-state current plotted against the normalized magnetic flux threading the $180^{\circ}$ connected ring strongly coupled to two 300 hydrogen atom chains serving as extended molecule lead portions that, in turn, are strongly coupled at their far ends to 2500 atoms chain driven lead sections (see main text for model details) using an energy buffer range of $W=0 \mathrm{eV}$ (full red line) and $W=5 \mathrm{eV}$ (full green line), compared to results obtained using the full system Hamiltonian spectrum (full black line). The applied bias voltage of $V_{b}=1 \mathrm{~V}$ and electronic temperature of $T=300 \mathrm{~K}$ are the same as those used to obtain the results presented in the main text. 
Fig. S5 presents the steady-state currents, calculated using the Sylvester equation (see Eq. (7) and appendix A in the main text) as a function of the normalized magnetic flux threading the $180^{\circ}$ connected ring strongly coupled to two 300 hydrogen atom chains serving as extended molecule lead portions that, in turn, are strongly coupled at their far ends to 2500 atoms chain driven lead sections (see main text for model details). Two energy cutoff windows of $1 \mathrm{eV}$ $(W=0 \mathrm{eV}$; full red line) and $11 \mathrm{eV}(W=5 \mathrm{eV}$; full green line) are considered. These correspond to total reduced Hamiltonian dimensions of 132 and 1,666, respectively. The result obtained using the full leads bandwidth and system dimensions (38 eV and 5,652 energy levels, respectively) are presented for comparison (full black line). Clearly, excluding from the calculation all states that reside strictly outside the $1 \mathrm{eV}$ Fermi transport window results in non-converged current estimations that deviate considerably from the full system bandwidth calculations throughout the $\mathrm{AB}$ period. Extending the cutoff window to $11 \mathrm{eV}$ provides sufficiently converged results while reducing the dimensionality of the calculation by a factor of 3.4. Based on this analysis, the value of $W=5 \mathrm{eV}$ was used to obtain all results presented in the main text. 


\section{Extended Molecule Size in Weakly Coupled Rings}

In molecular electronics calculations one often models an infinite system by a finite system subjected to appropriate boundary conditions. A useful approach to minimize the effect of the boundary conditions on the scattering region of interest is based on the notion of the extended-molecule. ${ }^{8-10}$ Here, the active molecule is augmented by portions of the lead sections, the sizes of which are set to obtain converged results. The choice of the geometry, chemistry, and size of the lead fragments taken to be part of the extended molecule region has also a crucial role when it comes to examining the coupling nature between the molecule and the leads. ${ }^{6,8}$

Nevertheless, in the weak lead-molecule coupling limit the energy levels associated with the active molecule are very sharp. This requires the inclusion of extremely large lead portions within the extended molecule section to avoid artificial effects resulting from the discrete nature of their spectrum. To demonstrate this, we plot in Fig. 5 the magnetic flux dependence of the current passing through the $104^{\circ}$ connected hydrogen ring, which is weakly coupled (see main text for model details) to hydrogen atomic chains that represent the extended molecule lead portions and are coupled on their far end to driven lead models. Two EM lead portion sizes are considered including 300 (full red line) and 600 (full green line) hydrogen atoms. The discrete spectrum of the 300 atoms EM lead portions is clearly manifested via strong current oscillations in the Aharonov-Bohm period. Doubling the EM lead portions size results in a reduction of the current oscillation amplitude but the discrete nature of their spectra remains evident.

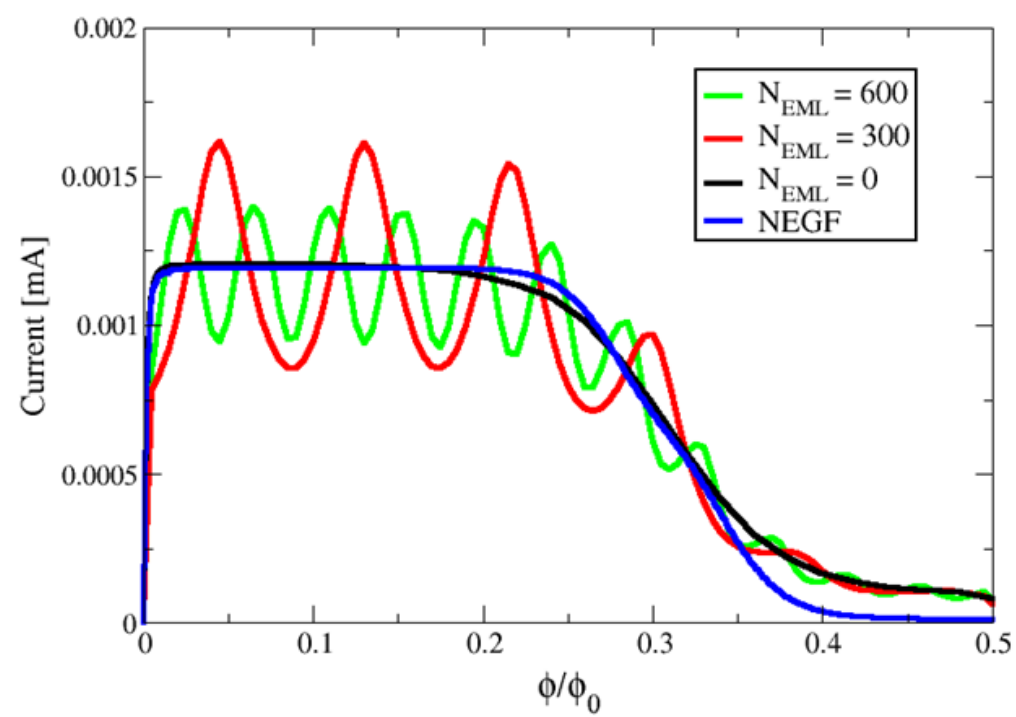

Figure S6: Steady-state current as a function of normalized magnetic flux threading the $104^{\circ}$ weakly connected hydrogen ring described in the main text with EM lead portions size of 0 (black), 300 (red), and 600 (green) hydrogen atoms, compared to the NEGF results (full blue line). The applied bias voltage of $V_{b}=1 \mathrm{~V}$ and electronic temperature of $T=300 \mathrm{~K}$ are the same as those used to obtain the results presented in the main text.

In principle, one could continue increasing the size of the EM lead portions until convergence of the current is achieved. Nevertheless, in the weak coupling limit, an efficient alternative would be to completely abandon the extended-molecule approach and couple the driven leads directly to the bare ring. As can be seen by the full black line in Fig. S6, this completely eliminates the current oscillations resulting from the discrete nature of the finite EM lead portions and provides results that are in fair agreement with non-equilibrium Green's functions calculations. This suggests that in the 
weak molecule-leads coupling limit, the boundary-conditions applied to the driven leads have a small effect on the scattering region even when they are directly coupled. Therefore, in this case the notion of the extended molecule, where the molecule is dressed by lead portions that buffer it from the boundary regions, becomes futile and may lead to unphysical behavior. We finally conclude that in a weakly coupled setup the molecule can (and probably should) be directly coupled to the driven leads, the energy spectra of which are appropriately broadened.

\section{$\underline{\text { References }}$}

(1) Zelovich, T.; Hansen, T.; Liu, Z.-F.; Neaton, J. B.; Kronik, L.; Hod, O. Parameter-Free Driven Liouville-von Neumann Approach for Time-Dependent Electronic Transport Simulations in Open Quantum Systems. J. Chem. Phys. 2017, 146 (9), 092331. https://doi.org/10.1063/1.4976731.

(2) Elenewski, J. E.; Gruss, D.; Zwolak, M. Communication: Master Equations for Electron Transport: The Limits of the Markovian Limit. J. Chem. Phys. 2017, 147 (15), 151101. https://doi.org/10.1063/1.5000747.

(3) Subotnik, J. E.; Hansen, T.; Ratner, M. A.; Nitzan, A. Nonequilibrium Steady State Transport via the Reduced Density Matrix Operator. J. Chem. Phys. 2009, 130 (14), 144105. https://doi.org/10.1063/1.3109898.

(4) Gruss, D.; Smolyanitsky, A.; Zwolak, M. Communication: Relaxation-Limited Electronic Currents in Extended Reservoir Simulations. J. Chem. Phys. 2017, 147 (14), 141102. https://doi.org/10.1063/1.4997022.

(5) Gruss, D.; Velizhanin, K. A.; Zwolak, M. Landauer's Formula with Finite-Time Relaxation: Kramers' Crossover in Electronic Transport. Sci. Rep. 2016, 6, 24514. https://doi.org/10.1038/srep24514.

(6) Zelovich, T.; Kronik, L.; Hod, O. Molecule-Lead Coupling at Molecular Junctions: Relation between the Real- and State-Space Perspectives. J. Chem. Theory Comput. 2015, 11 (10), 4861-4869. https://doi.org/10.1021/acs.jctc.5b00612.

(7) Zelovich, T.; Kronik, L.; Hod, O. Driven Liouville von Neumann Approach for Time-Dependent Electronic Transport Calculations in a Nonorthogonal Basis-Set Representation. J. Phys. Chem. C 2016, 120 (28), 15052-15062. https://doi.org/10.1021/acs.jpcc.6b03838.

(8) Moreira, R. A.; de Melo, C. P. On the Separability of the Extended Molecule: Constructing the Best Localized Molecular Orbitals for an Organic Molecule Bridging Two Model Electrodes. J Chem Phys 2014, 141 (12), 124712. https://doi.org/10.1063/1.4894845.

(9) Ryndyk, D. A.; Donarini, A.; Grifoni, M.; Richter, K. Many-Body Localized Molecular Orbital Approach to Molecular Transport. Phys. Rev. B 2013, 88 (8), 085404. https://doi.org/10.1103/PhysRevB.88.085404.

(10) Grigoriev, A.; Sköldberg, J.; Wendin, G.; Crljen, Ž. Critical Roles of Metal-Molecule Contacts in Electron Transport through Molecular-Wire Junctions. Phys. Rev. B 2006, 74 (4), 045401. https://doi.org/10.1103/PhysRevB.74.045401. 